\title{
RECONFIGURABLE SIBAR FILTERS WITH SIDEWALL ALUMINUM NITRIDE SIGNAL TRANSDUCTION
}

\author{
R. Tabrizian ${ }^{*}$ and F. Ayazi
}

School of Electrical and Computer Engineering, Georgia Institute of Technology, Atlanta, Georgia, USA

\section{ABSTRACT}

This paper reports, for the first time, on a versatile low-loss filter technology that can cover a wide frequency range $(30 \mathrm{MHz}$ to $3 \mathrm{GHz}$ ) and has electronic bandwidth and frequency tunability. The reconfigurable MEMS filters implemented using this technology have lithographically-defined frequencies, and use high- $Q$, low-motional-resistance silicon bulk acoustic resonators (SiBARs) with sidewall aluminum nitride (AlN) signal transduction. A proof-of-concept one-stage ladder filter at $\sim 100$ $\mathrm{MHz}$ implemented using this technology shows an insertion loss (IL) of $\sim 5 \mathrm{~dB}$ (with $50 \Omega$ termination) and a tunable bandwidth ranging between 110-360 kHz. An array of two adjacent channelselect filters with different bandwidths is also demonstrated.

\section{INTRODUCTION}

The fast growth of multiband and multifunction wireless communication systems has resulted in an increasing demand for a single-chip reconfigurable filter array. A few filter technologies with different levels of reconfigurability have been demonstrated to fulfill this demand, such as integrated tunable LCs [1] and evanescent mode cavity filters [2]. In these technologies, which are typically large in size, filter reconfiguration is mainly addressed by tuning the center frequency of a wideband filter using voltage tunable capacitors. Since filters implemented using these technologies are limited in their maximum achievable $Q$, they cannot be utilized to implement direct narrowband channel selection at RF to obviate subsequent down-conversion to baseband. Bulk acoustic wave (BAW) filters have been proposed to realize narrow bandwidth channel-select filters at RF [3-6]. However, since the resonance frequency of thickness-mode BAW devices (such as FBAR and SMR) is determined by thin films and substrate thicknesses and hence is nearly constant across the wafer [3-5], array of channel-select filters with frequencies covering the entire RF band cannot be realized without multiple film depositions, which makes their fabrication excessively complex and expensive. Solutions based on lateral-extensional resonators have the advantage of lithographically-defined frequencies; however, their application has been severely limited so far due to the low efficiency of electrostatic [6] and transverse piezoelectric $\left(d_{31}\right.$-based) transduction [7], which results in large motional impedances not suitable for construction of low loss filters.

\section{CONCEPT}

A truly reconfigurable filter technology should offer center frequency, bandwidth, and order reconfiguration in a wide frequency range. This requires a low-impedance, high- $Q$ resonator technology that can cover a useful spectrum via tuning and/or arraying (e.g., $30 \mathrm{MHz}$ to $3 \mathrm{GHz}$ ) and can lend itself well to electrical coupling to create arbitrary filter responses. This has been difficult to achieve using acoustic resonators due to various issues related to physical size, impedance, $Q$ factor and tuning. In our earlier work, we had reported on sidewall AIN transduction of high- $Q$ silicon bulk acoustic resonators (SiBAR) with rectangular bar geometry [8]. These are one-port width-extensional resonators with lithographically-defined frequencies. By engineering the dimensions, especially the thickness of the bar structures, lateral BAW resonators with very low motional resistances can be realized, enabled by the very efficient longitudinal piezoelectric coefficient $\left(d_{33}\right)$ of an AIN layer covering the large sidewalls of the device. Furthermore, this transduction configuration results in inherently negligible charge cancellation at the transduction port, since the metallic electrodes sandwiching the AlN layer are extended over uni-stress surfaces with uniformly-excited charge distribution (Fig. 1(a)). These resonators can essentially be interpreted as lateral FBARs sandwiching a layer of silicon with lithographically-defined dimensions. Following this interpretation, these devices not only inherit AIN FBAR advantages, but also surpass them in several aspects: since the resonance frequency of AlN-on-sidewall SiBARs is mainly defined by the lateral dimensions of their central silicon structure, bandpass filters with any desirable frequency can be implemented on a common substrate, while a single deposition of a piezoelectric AlN layer suffices for providing efficient electromechanical transduction to all the resonators. Furthermore, since these resonators have two dimensions with frequency dependencies extended at the two extremes (i.e., small frequency dependency on the length as opposed to high frequency sensitivity to the width of the resonator), a large range of frequencies can be realized, comfortably extending from $30 \mathrm{MHz}$ to $3 \mathrm{GHz}$ to cover the entire VHF and UHF bands. Last, but not least, real-time bandwidth and frequency tuning can be realized by running small currents through the silicon body of individual resonators. In this paper, we use these resonators in ladder-type topology to implement reconfigurable narrowband filters with extremely sharp skirts and low pass-band insertion loss.

\section{RECONFIGURABLE BANDPASS FILTER DESIGN}

A one-stage ladder-type filter consist of two AlN-on-sidewall SiBARs with high $Q(\sim 7,800$ in air $)$ and low motional resistance, one in series and one in parallel with offset frequency. Figure 1((b) and (c)) schematically shows the filter construction as well as the principle of operation.
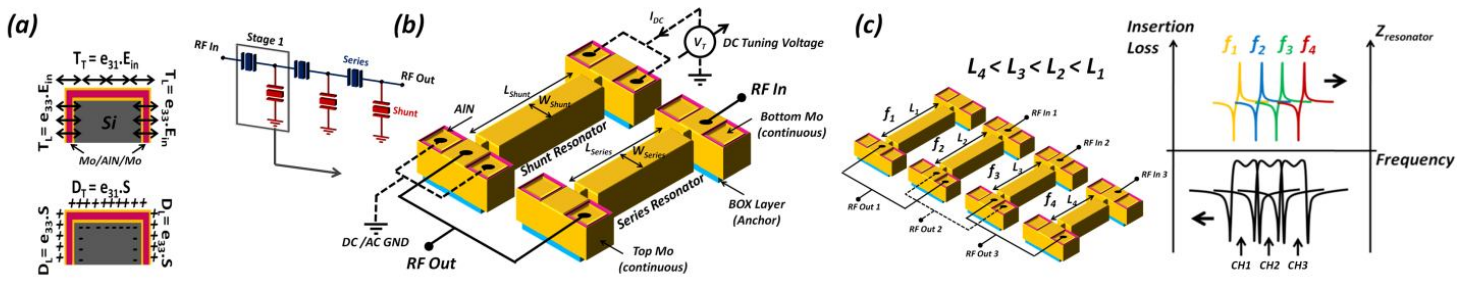

Figure 1: (a) The cross section of the resonator showing the actuation/sense mechanisms; (b) reconfigurable SiBAR filter with sidewall AlN transduction and thermal bandwidth and frequency tuning: one-stage ladder-type filter; (c) an array of adjacent channel-select ladder-type filters; each resonator serve as series and shunt element for two adjacent filters resulting in intrinsically-adjacent filter array. 
The small frequency offset and hence filter bandwidth is achieved by modifying the length of the SiBARs. Larger offsets can be obtained by adjusting the width of the resonator (primary frequency determining dimension). Furthermore, having a large negative TCF $\left(-32 \mathrm{ppm} /{ }^{\circ} \mathrm{C}\right)$, considerable frequency drift can be achieved by thermal tuning of each resonator of the filter, independently. This is done by passing a DC current via the body of the resonator resulting in the Joule heating. Large tuning ranges can be achieved using this technique by applying small DC voltages across the resonator.

\section{MEASUREMENT}

Figure 2 shows the SEM picture of the fabricated AlN-onsidewall SiBAR. The AC ground ports of this resonator can be used to apply tuning voltage across the resonator. Figure 3 shows the frequency response of two filters built using this technology as well as the configuration of the filters. Since the same resonator defines the pass-band transition of both filters, intrinsically adjacent bandpass channel-select filters can be implemented using this configuration. Figure 4 shows the thermal tuning characteristic of a single resonator. Large tuning ranges have been achieved with parabolic voltage dependency of resonance frequency. By applying this tuning technique to the shunt resonator of the ladder-type filter, significant bandwidth tuning is achieved. Figure 5 shows the frequency response of a one-stage ladder filter at $\sim 100 \mathrm{MHz}$ with IL $<5 \mathrm{~dB}$ (with $50 \Omega$ termination) and a tunable bandwidth ranged between 110-360 kHz. $227 \%$ of bandwidth tuning has been achieved with application of less than 2 volts.

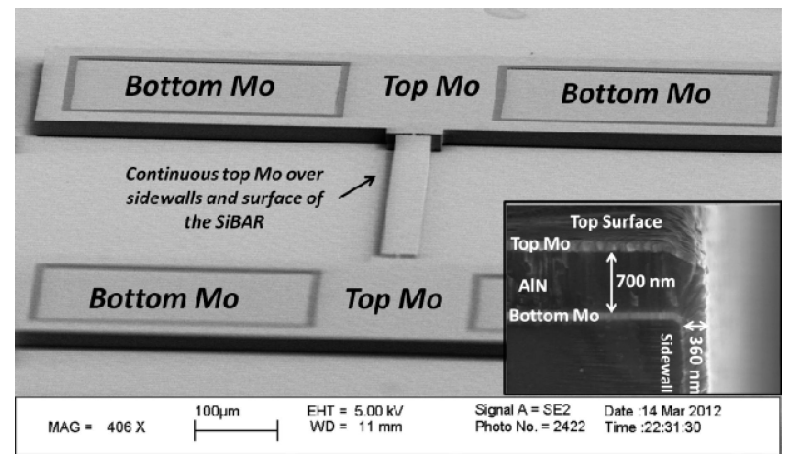

Figure 2: SEM picture of a SiBAR with sidewall AlN; the inset demonstrates the cross-section of these resonators showing the continuity of AlN and Mo films across the top surface and sidewalls of the SiBAR.

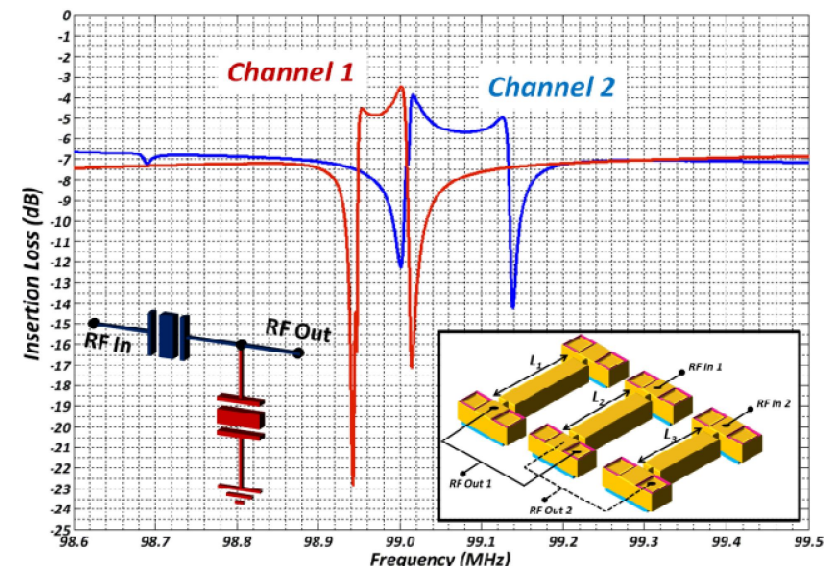

Figure 3: Frequency response of two adjacent filters; inset shows the configuration of these filters: the same resonator operates as the series and shunt resonator of channel 1 and 2 respectively.

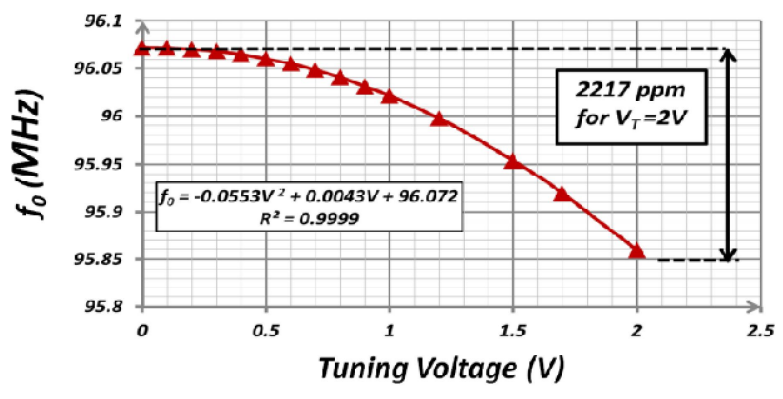

Figure 4: Frequency tuning characteristic of AlN-on-sidewall SiBAR showing large tuning achievable with small voltages and parabolic voltage dependency of the resonance frequency.

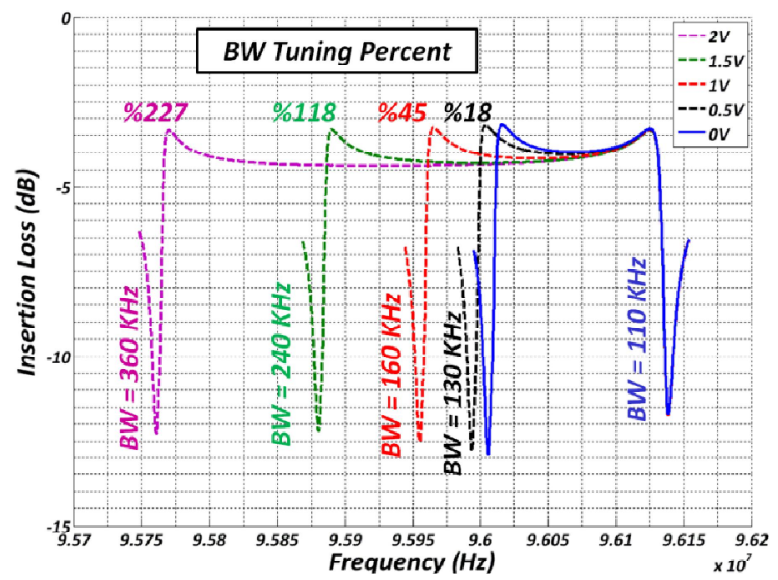

Figure 5: Bandwidth tunability for different voltages; the tuning will be done by passing current through the shunt resonator which results in its frequency decrease due to the SiBAR's negative TCF.

\section{ACKNOWLEDEMENT}

This work has been supported by National Science Foundation. Travel support has been generously provided by the Transducer Research Foundation.

\section{REFERENCES}

[1] M. Rais-Zadeh, et al., IEEE Journal of Microelectromechanical Systems, Volume 18, Issue 3, August 2009, pp. 942-949.

[2] H. Joshi, et al., IEEE MTT-S International Microwave Symposium Digest, June 2009, pp. 629-632.

[3] W. Pan and F. Ayazi, Tech. Digest 2008 IEEE International Frequency Control Symposium (IFCS 2008), Honolulu, HI, May 2008.

[4] K. M. Lakin, "Thin Film Resonators and High Frequency Filters," TFR Technologies, www.triquint.com.

[5] Lih Feng Cheow, et al., Solid State Sensor, Actuator and Microsystems Workshop (Hilton Head 2006), Hilton Head Island, South Carolina, June 4-8, 2006, pp. 304-307.

[6] S.-S. Li, et al., Int. Conf. on Solid-State Sensors, Actuators and Microsystems (Transducers), Lyon, France, June 2007. pp. 307-311.

[7] G. Piazza, et al., International Conference on Solid-State Sensors, Actuators and Microsystems (Transducers), Seoul, South Korea, June 2005. pp. 2065-2068.

[8] R. Tabrizian and F. Ayazi, Int. Conf. on Solid-State Sensors, Actuators and Microsystems (Transducers), Beijing, China, June 2011. pp. 1520-1523.

*R. Tabrizian, tel: +1-404-259-7322; roozbeh@gatech.edu 